\title{
COMPARAÇÃO ENTRE OS MÉTODOS PSICOFÍSICOS ESCALARES DE ESTIMAÇÃO DE MAGNITUDES E DE ESTIMAÇÃO DE CATEGORIAS DA PERCEPÇÃO SOCIAL DO ENFERMEIRO
}

\author{
Fátima Aparecida Emm Faleiros Sousa ${ }^{1}$ \\ Priscilla Hortense ${ }^{2}$ \\ Renata Alessandra Evangelista
}

Sousa FAEF, Hortense P, Evangelista RA. Comparação entre os métodos psicofísicos escalares de estimação de magnitudes e de estimação de categorias da percepção social do enfermeiro. Rev Latino-am Enfermagem 2004 setembro-outubro; 12(5):775-80.

O objetivo deste experimento foi escalonar a percepção social do enfermeiro pelos métodos de estimação de magnitudes e de categorias. Participaram estudantes do $2^{\circ}$ grau e universitários, profissionais atuantes e aposentados em medicina, psicologia, enfermagem e odontologia. Os resultados mostraram: (1) os traços asseado, responsável, limpo, cuidadoso e eficaz ocupam as primeiras posições em termos da percepção social do enfermeiro, enquanto os traços inútil, desonroso, desonesto, irresponsável e odioso ocupam as últimas posições nas escalas obtidas pelos dois métodos psicofísicos diretos e (2) o contínuo não-métrico de percepção social possui características protéticas.

DESCRITORES: percepção social; psicofísica; enfermagem

\section{COMPARISON BETWEEN THE PSYCHOPHYSICAL SCALE METHODS FOR MAGNITUDE ESTIMATION AND CATEGORY ESTIMATION OF NURSES' SOCIAL PERCEPTION}

This experiment aimed to rank nurses' social perception through the magnitude and category estimation methods. Study participants were high school and university students, active and retired professionals in the areas of medicine, psychology, nursing and dentistry. The results showed: (1) the characteristics neat, responsible, clean, careful and efficacious occupied the first positions in terms of nurses' social perception; whereas the characteristics useless, dishonorable, irresponsible and hateful occupied the last positions in the scales obtained by the two direct psychophysical methods, and (2) the non-metric social perception continuum has prosthetic characteristics.

DESCRIPTORS: social perception; psychophysics; nursing

COMPARACIÓN ENTRE LOS MÉTODOS PSICOFÍSICOS ESCALARES DE ESTIMACIÓN DE MAGNITUDES Y DE ESTIMACIÓN DE CATEGORIAS DE LA PERCEPCIÓN SOCIAL DEL ENFERMERO

El objetivo de este experimento fue escalonar la percepción social del enfermero por los métodos de estimación de magnitudes y de categorías. Participaron alumnos de la enseñanza fundamental y de universidades, profesionales actuantes y jubilados en medicina, psicología, enfermería y odontología. Los resultados mostraron que (1) las características aseado, responsable, limpio, cuidadoso y eficaz ocupan las primeras posiciones en términos de la percepción social del enfermero, como las características inútil, deshonroso, deshonesto, irresponsable y odioso ocupan las últimas posiciones en las escalas obtenidas por los dos métodos psicofísicos directos y (2) el continuo no métrico de percepción social tiene características protéticas.

DESCRIPTORES: percepción social; psicofísica; enfermería

\footnotetext{
${ }^{1}$ Professor Associado, e-mail: faleiros@eerp.usp.br; ${ }^{2}$ Mestranda. Escola de Enfermagem de Ribeirão Preto, da Universidade de São Paulo, Centro Colaborador da OMS para o desenvolvimento da pesquisa em enfermagem; ${ }^{3}$ Docente da Universidade de Araraquara
} 


\section{INTRODUÇÃO}

$E_{\mathrm{m}}$ um estudo sobre a percepção social do enfermeiro ${ }^{(1)}$, o autor teve como objetivo escalonar a percepção social do enfermeiro pelo método psicofísico de estimação de categorias. Para tal, foram convidados estudantes universitários das áreas de psicologia, enfermagem, odontologia e medicina. Os resultados mostraram: 1) em nosso meio o traço asseado exerce papel de centralidade, o qual, juntamente com o traço responsável, serve como estereótipo do enfermeiro e 2) as altas correlações entre as estimativas de categorias dos subgrupos refletem grande concordância nos graus de atribuições, dados pelas diferentes amostras de estudantes universitários aos traços que caracterizam o profissional enfermeiro, indicando que as estimativas são estáveis entre as amostras.

No contexto desse estudo, traço foi considerado como aquele atribuído ao enfermeiro e que pode ser resultante de inferência baseada em observações anteriores, do que muitas pessoas dizem, no dia-a-dia, e também de leituras eventuais que foram feitas acerca desse profissional.

O estudo da percepção social de determinada profissão identifica traços (adjetivos) que são atribuídos a essa profissão na sociedade em que vivemos e o quão freqüente são usados na vida cotidiana. Neste estudo será realizada a investigação sobre a percepção social do enfermeiro através de procedimentos relativos à psicofísica sensorial.

Em um outro estudo ${ }^{(2)}$, a autora escalonou os adjetivos atribuídos à dor pós-operatória através de dois métodos psicofísicos, a estimação de magnitudes e o emparelhamento intermodal com a modalidade de resposta comprimento de linhas. Os resultados obtidos foram que: 1) independente do método psicofísico utilizado, os adjetivos aniquilante, alucinante, colossal e fulminante foram considerados aqueles de maior atribuição para a dor pós-operatória e os adjetivos insignificante, leve, discreta e pequena foram considerados os de menor atribuição; 2) as ordenações resultantes dos dois métodos produzem posições de atribuição à dor pós-operatória altamente concordantes para os diferentes adjetivos; 3 ) a escala de atribuição da dor pós-operatória é válida, estável e consistente, já que o expoente obtido através do emparelhamento por comprimento de linhas e estimação de magnitudes não foi significativamente diferente do expoente predito por Stevens ${ }^{(3)}$.
O método de estimação de magnitude é um tipo de emparelhamento que consiste em levar os sujeitos a designarem valores numéricos (escores) aos estímulos apresentados pelo pesquisador, de forma a equipará-los aos números. Com o uso desse método, a razão entre os números atribuídos reflete a razão entre as respostas (intensidades percebidas) dos sujeitos aos estímulos que estão sendo julgados ${ }^{(3)}$.

Os métodos de mensuração (estimação de magnitudes, estimação de categorias e emparelhamento intermodal) e a lei psicofísica têm sido usados por alguns pesquisadores em enfermagem com o propósito de determinar um índice de respostas ou de conceitos subjetivos dos enfermeiros e clientes hospitalizados ou não(4).

A estratégia psicofísica é superior a inúmeras outras estratégias comumente utilizadas em Enfermagem por fornecer informações quantitativas e qualitativas sobre a intensidade dos julgamentos dos pacientes, médicos e enfermeiras ${ }^{(5-6)}$.

Podemos julgar tanto a qualidade quanto a quantidade de um dado estímulo. Por exemplo, podemos julgar tanto a qualidade quanto a quantidade de um som como altas ou baixas, ou seja, suas qualidades; e também julgar a intensidade sonora de um estímulo, isto é, os graus de magnitude ou quantidade. Assim, quando julgamos a qualidade devemos pensar num contínuo metatético e quando julgamos a quantidade, num contínuo protético. As intensidades dos estímulos têm sido denominadas de protético porque grande quantidade delas é baseada em processos fisiológicos em que a nova excitação é adicionada à excitação já existente. De outro lado, um processo de substituição parece estar subjacente aos contínuos denominados metatéticos ${ }^{(3)}$.

O método de estimação de magnitudes também foi usado com sucesso na mensuração da gravidade de diferentes quadros clínicos ${ }^{(7)}$. Os autores utilizaram 0 método de estimação de magnitudes e emparelhamento intermodal com o objetivo de escalonar a gravidade de quadros clínicos cirúrgicos por médicos e enfermeiros. Os resultados mostraram que: 1) os quadros clínicos cirúrgicos de aneurisma de aorta, aneurisma cerebral e revascularização do miocárdio foram considerados aqueles de maior gravidade, enquanto que os quadros clínicos cirúrgicos adenoidectomia, amigdalectomia e curetagem uterina os de menor gravidade, todos obtidos através dos diferentes métodos psicofísicos diretos; 2) a escala de razão de gravidade, derivada do emparelhamento por 
comprimento de linhas e forças dinamométricas, não foi significativamente diferente do que o expoente estabelecido $(0,77)$ pela transitoriedade das escalas.

\section{OBJETIVOS}

- comparar as escalas derivadas de julgamentos intervalares (estimativas de categorias) com as escalas derivadas de julgamentos de razão (estimativas de magnitudes);

- verificar, através da comparação entre as estimativas de magnitudes e as estimativas de categorias, se o contínuo não-métrico de percepção social tem características protéticas ou metatéticas;

- verificar se as ordenações dos graus de percepção social derivadas dos dois métodos psicofísicos são similares entre $\mathrm{si}$;

- verificar a estabilidade e/ou equivalência das escalas de categorias e de razão produzidas através de duas modalidades de respostas diferentes, quais sejam numérica sem limites (estimativas de magnitudes) e numérica com limites (estimativas de categorias).

\section{MÉTODO}

Participantes

Os sujeitos foram selecionados aleatoriamente, sendo que participaram 204, sendo 56 estudantes das segundas e terceiras séries do $2^{\circ}$ grau; 44 estudantes universitários dos cursos de graduação em enfermagem, medicina, psicologia e odontologia, 56 profissionais atuantes, sendo eles enfermeiros, médicos, psicólogos e dentistas e 48 profissionais aposentados em enfermagem, medicina, psicologia e odontologia, sendo os profissionais atuantes e aposentados de diferentes especialidades. Todos oriundos da cidade de Ribeirão Preto, com idades variando entre 18 e 75 anos e eram ingênuos, ou seja, os sujeitos não tinham experiência com experimentos dessa natureza.

Material

Foram elaborados 2 blocos de papel contendo, na primeira página, instruções específicas para cada tipo de método psicofísico e nas páginas seguintes uma lista de 15 traços de personalidade resultantes de um estudo ${ }^{(1)}$ e respectivas definições.

Procedimento

Para a realização deste trabalho, selecionou-se de um estudo ${ }^{(1)}, 15$ adjetivos que caracterizam o profissional enfermeiro em nossa sociedade, sendo 5 de maior, 5 de grau neutro e 5 de menor atribuição, sendo que esses adjetivos foram escalonados pelo método de estimação de categorias. Esses adjetivos selecionados foram escalonados pelos métodos de estimação de magnitudes e de emparelhamento intermodal, envolvendo o contínuo de resposta comprimento de linhas, além do método de estimação de categorias.

Foram utilizados o método de estimação em categorias e o método de estimação de magnitudes. No procedimento utilizado no primeiro método, qual seja, estimação em categorias, a tarefa dos participantes consistia em assinalar um escore, o qual variava de 0 a 6 , a cada traço em função da sua pertinência para caracterizar o enfermeiro em nossa sociedade. O participante foi instruído a assinalar ao traço de maior atribuição o escore máximo de 6 e ao de menor, o escore mínimo de 0 . Os outros escores intermediários de 1 a 5 deveriam ser utilizados para indicar graus intermediários de utilização que os traços possuem para caracterizar o enfermeiro. Previamente não foram estabelecidos os traços de atribuição máxima e mínima, sendo que cada participante estabeleceu apenas 15 estimativas, sendo uma para cada traço de personalidade selecionado.

No segundo método, a tarefa dos participantes foi assinalar um número a cada traço que fosse proporcional à quantidade de definição que aquele adjetivo tem em relação ao profissional enfermeiro em nossa sociedade. Dessa forma, se o participante julgasse que um dado adjetivo define duas vezes mais o enfermeiro do que um outro adjetivo, ele deveria assinalar a ele um número duas vezes maior. Se ele julgasse que um dado adjetivo possui metade da definição do enfermeiro do que um outro adjetivo, ele deveria assinalar um número que fosse metade do atribuído àquele adjetivo. Os diferentes adjetivos foram apresentados em uma série de quinze, os quais foram dispostos, um a um, escritos em cartões separados, numa ordem totalmente aleatória para cada participante. Cada participante estabeleceu 15 estimativas, sendo uma para 
cada adjetivo. Estímulo padrão e módulo não foram previamente designados.

As amostras foram independentes, tendo sido utilizados 204 participantes, sendo 56 estudantes do $2^{\circ}$ grau, dos quais 28 julgaram pelo método de estimação de categorias e 28 julgaram pelo método de estimação de magnitudes; 44 estudantes universitários, dos quais 22 julgaram pelo método de estimação de categorias e 22 julgaram pelo método de estimação de magnitudes; 56 profissionais atuantes, dos quais 28 julgaram pelo método de estimação de categorias e 28 julgaram pelo método de estimação de magnitudes e 48 profissionais aposentados, dos quais 24 julgaram pelo método de estimação de categorias e 24 julgaram pelo método de estimação de magnitudes.
O experimento foi realizado num laboratório e os sujeitos fizeram os julgamentos individualmente.

\section{RESULTADOS E DISCUSSÃO}

As estimativas médias para os diferentes traços realizadas pelos diferentes grupos de participantes (A1 amostra composta por estudantes universitários, A2 por profissionais atuantes, A3 por profissionais aposentados e A4 por estudantes do $2^{\circ}$ grau) e as respectivas ordenações de posições estão apresentadas abaixo (Tabelas 1 e 2), para os métodos psicofísicos utilizados: Estimação de Categorias (EC) e Estimação de Magnitudes (EM).

Tabela 1 - Média aritmética das estimativas de categorias (EC), e ordenação das posições (OP) de atribuição julgadas de cada traço das diferentes amostras (A1, A2, A3 e A4)

\begin{tabular}{|c|c|c|c|c|c|c|c|c|}
\hline & \multicolumn{2}{|c|}{ A 1} & \multicolumn{2}{|c|}{ A 2} & \multicolumn{2}{|c|}{ A 3} & \multicolumn{2}{|c|}{ A 4} \\
\hline Traços & EC & OP & EC & OP & EC & OP & EC & $\mathrm{OP}$ \\
\hline Asseado & 5,22 & $1^{\circ}$ & 5,47 & $1^{\circ} \stackrel{0}{1}$ & 5,86 & $4 \stackrel{\circ}{2}$ & 5,80 & $1^{\circ}=$ \\
\hline Responsável & 4,81 & $4^{\circ}$ & 5,34 & $2^{\circ}$ & 6,00 & $2^{\circ}$ & 5,69 & 30 \\
\hline Limpo & 4,89 & $3^{\circ}$ & 5,00 & $4 \stackrel{\circ}{2}$ & 6,00 & $1 \stackrel{\circ}{1}$ & 5,76 & $2^{\circ}$ \\
\hline Cuidadoso & 4,93 & $2^{\circ}$ & 5,24 & $3 \stackrel{0}{2}$ & 5,95 & $3 \stackrel{0}{2}$ & 5,66 & 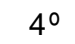 \\
\hline Eficaz & 4,74 & $5^{\circ}$ & 4,93 & $5^{0}$ & 5,76 & $5^{\circ}$ & 5,41 & $5^{\circ}$ \\
\hline Insatisfeito & 2,81 & $8^{\circ}$ & 3,03 & $7^{\circ} \stackrel{0}{1}$ & 1,38 & $10^{\circ}$ & 1,21 & $10^{\circ}$ \\
\hline Letrado & 3,04 & $7^{\circ}$ & 3,00 & $8^{\circ}$ & 5,62 & $6^{\circ}$ & 5,28 & $8^{\circ}$ \\
\hline Melindroso & 3,44 & $6^{\circ}$ & 3,14 & 60 & 5,38 & $8^{\circ}$ & 5,31 & $7^{\circ}$ \\
\hline Pensativo & 2,37 & $9^{\circ}$ & 2,03 & $10^{\circ}$ & 5,48 & $7^{\circ}$ & 5,41 & $6^{\circ}$ \\
\hline Temperamental & 2,19 & $10^{\circ}$ & 2,10 & 90 & 2,62 & 90 & 3,86 & $9^{\circ}$ \\
\hline Odioso & 0,70 & $12^{\circ}$ & 0,48 & $13^{\circ}$ & 0 & $13^{\circ}$ & 0,31 & $11^{\circ}$ \\
\hline Irresponsável & 0,93 & $11^{\circ}$ & 0,34 & $14^{\circ}$ & 0 & $15^{\circ}$ & 0,07 & $15^{\circ}$ \\
\hline Desonesto & 0,41 & $14^{\circ}$ & 0,66 & $11^{\circ}$ & 0 & $14^{\circ}$ & 0,24 & $12^{\circ}$ \\
\hline Desonroso & 0,59 & $13^{\circ}$ & 0,34 & $15^{\circ}$ & 0,05 & $11^{\circ}$ & 0,21 & $13^{\circ}$ \\
\hline Inútil & 0.15 & $15^{\circ}$ & 0,52 & $12^{\circ}$ & 0,05 & $12^{\circ}$ & 0,10 & 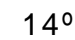 \\
\hline
\end{tabular}

Tabela 2 - Média geométrica das estimativas de magnitudes (EM) e ordenação das posições (OP) de atribuição julgadas de cada traço das diferentes amostras (A1, A2, A3 e A4)

A 1

\begin{tabular}{|c|c|c|c|c|c|c|c|c|}
\hline Traços & EM & OP & EM & OP & EM & OP & EM & OP \\
\hline Asseado & 29,09 & $2^{\circ}$ & 15,64 & $3^{0}$ & 14,96 & 10 & 16,53 & $2^{\circ}$ \\
\hline Responsável & 27,75 & 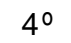 & 15,77 & $2^{\circ}$ & 13,45 & 30 & 17,45 & $1 \stackrel{\circ}{2}$ \\
\hline Limpo & 32,11 & $1 \stackrel{\circ}{\circ}$ & 15,05 & $4^{\circ}$ & 13,97 & $2^{\circ}$ & 14,70 & $5^{\circ}$ \\
\hline Cuidadoso & 28,08 & $3 \stackrel{0}{0}$ & 16,08 & 10 & 13,11 & $4 ㅇ$ & 15,22 & $4 \stackrel{0}{-1}-10$ \\
\hline Eficaz & 26,85 & $5^{\circ}$ & 14,26 & $5^{\circ}$ & 10,98 & $5^{\circ}$ & 15,46 & $3^{\circ}$ \\
\hline Insatisfeito & 12,51 & $10^{\circ}$ & 6,90 & $6^{\circ}$ & 5,90 & $6^{\circ}$ & 4,62 & 90 \\
\hline Letrado & 18,33 & 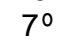 & 2,44 & $10^{\circ}$ & 1,87 & $10^{\circ}$ & 4,67 & $8^{\circ}$ \\
\hline Melindroso & 25,04 & 60 & 5,85 & 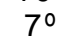 & 4,32 & $7^{\circ}$ & 8,42 & $6^{\circ}$ \\
\hline Pensativo & 12,91 & $9 \circ$ & 3,18 & $8^{\circ}$ & 3,76 & $8^{\circ}$ & 6,54 & 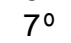 \\
\hline Temperamental & 15,41 & $8^{\circ}$ & 3,01 & 90 & 3,33 & 90 & 4,06 & $10^{\circ}$ \\
\hline Odioso & 2,30 & $12^{\circ}$ & 1,00 & $13^{\circ}$ & 1,00 & $13^{\circ}$ & 1,11 & $11^{\circ}$ \\
\hline Irresponsável & 2,89 & $11^{\circ}$ & 1,07 & $11^{\circ}$ & 1,11 & $11^{\circ}$ & 1,08 & $12^{\circ}$ \\
\hline Desonesto & 2,23 & $13^{\circ}$ & 1,00 & $14^{\circ}$ & 1,00 & $14^{\circ}$ & 1,00 & $14^{\circ}$ \\
\hline Desonroso & 2,23 & $14^{\circ}$ & 1,00 & $15^{\circ}$ & 1,00 & $15^{\circ}$ & 1,00 & $15^{\circ}$ \\
\hline Inútil & 1,61 & $15^{\circ}$ & 1,06 & $12^{\circ}$ & 1,05 & $12^{\circ}$ & 1,07 & $13^{\circ}$ \\
\hline
\end{tabular}

Pode-se observar, nessas tabelas, que, independente do método psicofísico utilizado, os traços asseado, responsável, limpo, cuidadoso e eficaz foram aqueles de maior atribuição enquanto que os traços inútil, 
desonesto, desonroso, irresponsável e odioso foram os de menor atribuição. De fato, o coeficiente de concordância de Kendall (W) mostrou que as diferentes amostras foram altamente concordantes quanto à ordenação desses diferentes traços. Esses valores do coeficiente de concordância foram $\mathrm{W}=0,93$ para o método de $E C, W=0,95$ para o de $E M$, sendo todos altamente significativos $(p<0,001)$.

Como conseqüência dessa alta concordância dos julgamentos feitos pelas diferentes amostras, as estimativas de todos os participantes foram agrupadas em separado para cada método e suas respectivas ordenações de posições (Tabela 3). Pode-se observar nitidamente que essas ordenações, considerando-se cada método, não foram substancialmente diferentes daquelas produzidas pelas diferentes amostras (Tabelas 1 e 2 ).

Asseado, responsável, limpo, cuidadoso e eficaz foram aqueles traços (adjetivos) de maior atribuição e inútil, desonroso, desonesto, irresponsável e odioso os de menor atribuição ${ }^{(1)}$.

Um coeficiente de concordância, aplicado às estimativas efetuadas por meio de cada método para os quinze adjetivos, mostrou $\mathrm{W}=0,96(\mathrm{p}<0,001)$, indicando, portanto, que a ordenação por postos derivada das estimativas de cada método é altamente concordante. Por exemplo, o adjetivo inútil ocupa a 15a posição e o adjetivo responsável ocupa a $2^{\underline{a}}$ posição nos dois contínuos de respostas. A análise que se segue foi feita considerandose as estimativas médias (Tabela 3 ).

Tabela 3 - Média aritmética das estimativas de categorias (EC), média geométrica das estimativas de magnitudes (EM) e ordenação das posições (OP) de atribuição, julgadas para cada traço considerando todas as amostras juntas

\begin{tabular}{|c|c|c|c|c|}
\hline Traços & EC & OP & EM & OP \\
\hline Asseado & 5,57 & 10 & 17,76 & $1^{0}$ \\
\hline Responsável & 5,42 & $2^{\circ}$ & 17,46 & $2^{\circ}$ \\
\hline Limpo & 5,36 & $4^{\circ}$ & 17,05 & $3^{\circ}$ \\
\hline Cuidadoso & 5,40 & $3^{0}$ & 16,83 & $4^{\circ}$ \\
\hline Eficaz & 5,09 & $5^{\circ}$ & 15,53 & $5^{0}$ \\
\hline Insatisfeito & 2,15 & $10^{\circ}$ & 6,67 & 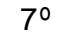 \\
\hline Letrado & 4,16 & $7^{\circ}$ 은 & 4,10 & $10^{\circ}$ \\
\hline Melindroso & 4,25 & $6^{\circ}$ & 8,03 & $6^{0}$ \\
\hline Pensativo & 3,70 & $8^{\circ}$ & 5,34 & $8^{0}$ \\
\hline Temperamental & 2,67 & $9^{\circ}$ & 4,62 & $9^{\circ}$ \\
\hline Odioso & 0,43 & $11^{\circ}$ & 1,21 & $12^{\circ}$ \\
\hline Irresponsável & 0,35 & $12^{0}$ & 1,32 & $11^{\circ}$ \\
\hline Desonesto & 0,35 & $13^{\circ}$ & 1,17 & $13^{\circ}$ \\
\hline Desonroso & 0,31 & $14^{\circ}$ & 1,17 & $14^{\circ}$ \\
\hline Inútil & 0,22 & $15^{\circ}$ & 1,15 & $15^{\circ}$ \\
\hline
\end{tabular}

Para verificar se o contínuo de percepção social tem características protéticas ou metatéticas, como ocorre com os contínuos sensoriais aditivos, as médias aritméticas das estimativas de categorias foram projetadas em função das médias geométricas das estimativas de magnitudes. Em coordenadas lineares a relação entre essas estimativas (estimativas de categorias $x$ estimativas de magnitudes) mostrou leve concavidade descendente. Também, quando as médias aritméticas das estimativas de categorias foram projetadas em função dos logaritmos das médias geométricas das estimativas de magnitudes, a relação mostrou concavidade ascendente. A Figura 1 mostra claramente essa relação em coordenadas lineares e a Figura 2 em coordenadas monologarítmicas. Tomadas juntas, fica demonstrado que o contínuo de percepção social tem características de contínuo protético ou aditivo.

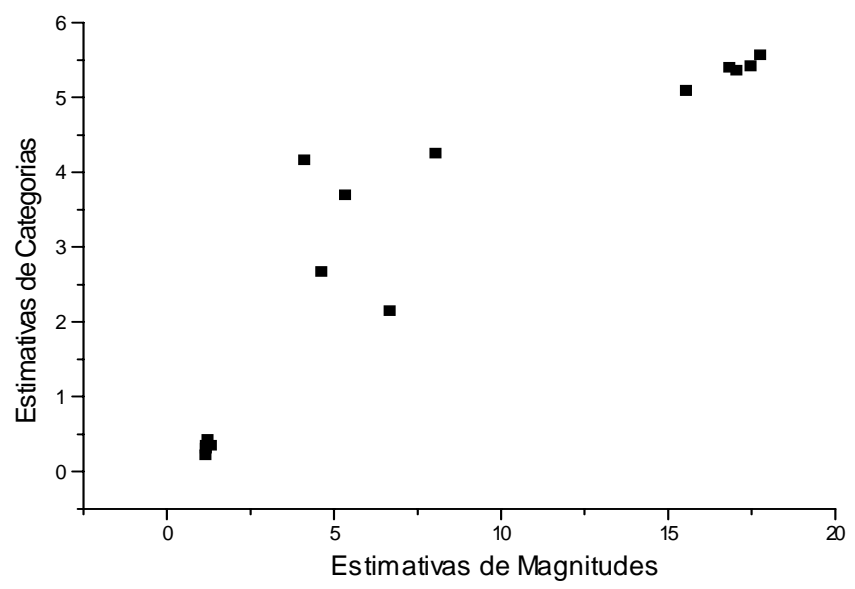

Figura 1 - Relação entre as médias aritméticas das estimativas de categorias e as médias geométricas das estimativas de magnitudes da percepção social do enfermeiro em coordenadas lineares

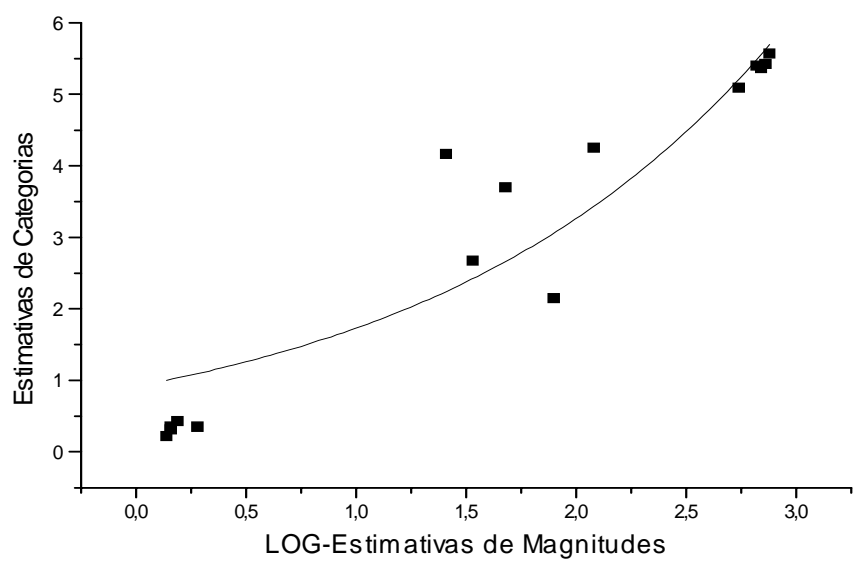

Figura 2 - Relação entre as médias aritméticas das estimativas de categorias e os logaritmos das médias geométricas das estimativas de magnitudes da percepção social do enfermeiro 


\section{CONCLUSÕES}

Os dados do Experimento realizado neste estudo permitem concluir que:

- os traços asseado, responsável, limpo, cuidadoso e eficaz ocupam as primeiras posições em termos da percepção social do enfermeiro, enquanto os traços inútil, desonroso, desonesto, irresponsável e odioso ocupam as últimas posições nas escalas obtidas pelos dois métodos psicofísicos diretos;

- as ordenações resultantes dos dois métodos produzem posições de percepção social do enfermeiro altamente concordantes para os diferentes adjetivos;

- o contínuo não-métrico de percepção social possui características protéticas.

\section{REFERÊNCIAS BIBLIOGRÁFICAS}

1. Sousa FAEF. Percepção social do enfermeiro. Rev Latinoam Enfermagem 2000 janeiro; 8(1):31-4.

2. Sant'Ana RP, Hortense P, Giuntini PB, Faleiros Sousa FAE. Validation of the ratio scale of postoperative pain descriptors through the cross-modality matching method. In: Da Silva JA, Matsushima EH, Ribeiro NP Filho, organizadores. Eighteenth Annual Meeting of the International Society for PsychophysicsFechner Day 2002. Rio de Janeiro (RJ): Ed. Legis Summa; 2002. p. 514-9.

3. Stevens SS. Psychophysics: introduction to its perceptual, neural and social prospects. In: Stevens G, organizador. New York (USA): Wiley; 1975.

4. Faleiros Sousa FAE. Mensuração de atributos sociais e clínicos: um enfoque experimental. Ribeirão Preto-SP: FUNPEC Editora; 2000.

5. Sennot-Miller L, Murdaugh C, Hinshaw AS. Magnitude estimation: issues and practical application. Western J Nurs Res 1988; 10:414-4.

6. Meek PM, Sennot-Miller L, Ferketich SL. Scaling stimuli with magnitude estimation. Res Nurs \& Health 1992; 15:7781.

7. Faleiros Sousa FAE, Sant'Ana RP, Evangelista RA, Lessa SS. Scaling Seriousness of the Clinical-Surgical IIIness. In: Da Silva JA, Matsushima EH, Ribeiro NP Filho, organizadores. Eighteenth Annual Meeting of the International Society for Psychophysics-Fechner Day 2002. Rio de Janeiro (RJ):Ed. Legis Summa; 2002. p. 535-8. 